\title{
Integrative Oncology
}

\section{Cancer Cachexia: Traditional Therapies and Novel Molecular Mechanism-Based Approaches to Treatment}

\author{
Nagi B. Kumar, PhD, RD, FADA* \\ Aslam Kazi, PhD \\ Tiffany Smith, BS \\ Theresa Crocker, MS, RD \\ Daohai Yu, PhD \\ Richard R. Reich, PhD \\ Kiran Reddy, MD \\ Sally Hastings, RD \\ Martine Exterman, MD \\ Lodovico Balducci, MD \\ Kyle Dalton, BS \\ Gerold Bepler, MD
}

\author{
Address \\ *Department of Population Sciences, H. Lee Moffitt Cancer Center \& \\ Research Institute at the University of South Florida College of Medicine, \\ 12902 Magnolia Drive, Tampa, FL 33612, USA \\ E-mail: nagi.kumar@moffitt.org \\ ๑ The Author(s) 2010
}

\section{Opinion statement}

The complex syndrome of cancer cachexia (CC) that occurs in 50\% to $80 \%$ cancer patients has been identified as an independent predictor of shorter survival and increased risk of treatment failure and toxicity, contributing to the mortality and morbidity in this population. CC is a pathological state including a symptom cluster of loss of muscle (skeletal and visceral) and fat, manifested in the cardinal feature of emaciation, weakness affecting functional status, impaired immune system, and metabolic dysfunction. The most prominent feature of $\mathrm{CC}$ is its non-responsiveness to traditional treatment approaches; randomized clinical trials with appetite stimulants, 5-HT3 antagonists, nutrient supplementation, and Cox-2 inhibitors all have failed to demonstrate success in reversing the metabolic abnormalities seen in CC. Interventions based on a clear understanding of the mechanism of CC, using validated markers relevant to the underlying metabolic abnormalities implicated in $\mathrm{CC}$ are much needed. Although the etiopathogenesis of $\mathrm{CC}$ is poorly understood, studies have proposed that $\mathrm{NFkB}$ is upregulated in $\mathrm{CC}$, modulating immune and inflammatory responses induce the 
cellular breakdown of muscle, resulting in sarcopenia. Several recent laboratory studies have shown that $n-3$ fatty acid may attenuate protein degradation, potentially by preventing NFkB accumulation in the nucleus, preventing the degradation of muscle proteins. However, clinical trials to date have produced mixed results potentially attributed to timing of interventions (end stage) and utilizing outcome markers such as weight which is confounded by hydration, cytotoxic therapies, and serum cytokines. We propose that selective targeting of proteasome activity with a standardized dose of omega-3-acid ethyl esters, administered to cancer patients diagnosed with early stage $\mathrm{CC}$, in addition to a standard intervention with nutritionally adequate diet and appetite stimulants, will alter metabolic abnormalities by downregulating NFkB, preventing the breakdown of myofibrillar proteins and resulting in increasing serum protein markers, lean body mass, and functional status.

\section{Introduction}

The complex syndrome of cancer cachexia (CC) is a major contributor to the mortality and morbidity of cancer patients $[1,2 \bullet, 3 \bullet, 4,5]$. CC is a pathological state where loss of muscle (skeletal and visceral) or muscle and fat occurs, manifested in the cardinal feature of emaciation, weakness affecting functional status, impaired immune system and metabolic dysfunction $[1,2 \bullet, 3 \bullet, 4,5]$. CC occurs in $50 \%$ to $80 \%$ cancer patients and identified as an independent predictor of shorter survival and increases the risk of treatment failure and toxicity in this population $[1,2 \bullet$, $3 \bullet, 4,5]$. In 2006, it was estimated that over 5 million individuals in the US suffered from cachexia because of cancer or other conditions. CC continues to be an important problem in clinical oncology [5].

\section{Pathogenesis}

- Although the etiopathogenesis of CC is poorly understood, multiple biologic pathways are known to be involved, including pro-inflammatory cytokines [6-8], neuroendocrine hormones [4, 9•], downregulation of IGF-1 [2•] and tumor-specific factors such as proteolysis inducing factor (PIF) $[10,11 \bullet \bullet$. Cytokines that are important in the initiation of the acute phase response (APR) have also been hypothesized in the genesis of CC $[12 \bullet \bullet, 13 \bullet]$ or the result rather than the cause of CC. Interleukin-6 (IL-6), may play a role in muscle wasting in certain animal tumors, possibly through both lysosomal (cathepsin) and non-lysosomal (proteasome) pathways [14]. TNF-alpha has been shown to have direct catabolic effect on skeletal muscle and adipose tissue and produces muscle atrophy through a depression of protein synthesis and an increase in protein degradation through the ubiquitin-proteasome proteolytic pathway, involving the formation of reactive oxygen species leading to upregulation of the transcription factor-NF-kB $[15,16]$. NF-kB is a known regulator of the genes that encode cytokines, cytokine receptors, cell-adhesion molecules, modulating immune and inflammatory responses by increasing production of cytokines that induce proteolysis and breakdown of myofibrillar proteins [15-19]. The most plausible evidence thus comes from animal studies that have demonstrated that etiopathogenesis of CC may be contributed by increasing proteolysis and breakdown of myofibrillar proteins and degradation by the proteasome. 


\section{Presentation}

- Common clinical manifestations of CC are muscle wasting, unplanned weight loss, anorexia, anemia, and altered immune function which contribute to increased fatigue, disability, and diminished quality of life. The most prominent feature of CC is its non-responsiveness to traditional treatment approaches; randomized clinical trials with appetite stimulants [5, 20-25], nutrient supplementation [26, 27], 5-HT3 antagonists [28, 29], and Cox-2 inhibitors [30••] all have failed to demonstrate success in reversing the metabolic abnormalities seen in CC. More recently, staging guidelines have been proposed $[31,32 \bullet]$ for CC, indicating that CC represents a spectrum initially observed as unplanned weight loss (pre-cachexia) progressing through degrees of severity (four stages) to the point where the cancer patient is depleted of both muscle and fat, immunocompromised and will eventually die of complications from these issues [33].

\section{Prognosis}

- Cancer cachexia has been identified as an independent predictor of shorter survival and increases the risk of treatment failure and toxicity, interruption of serial treatment and survival in this population $[1,2 \bullet, 3 \bullet$, $4,5]$. CC is much more difficult to treat in the elderly and the consequences of failure to correct malnutrition delays recovery and has a significant impact on functional dependence and quality of life in this patient population $[33,34 \bullet \bullet]$.

\section{Treatment}

- Over the years, since one of the features identified in pre-cachexia is loss of appetite and reduced food intake, CC was thus considered a nutritional disorder seen in advanced cancer patients. Hence, it was logical to infer that traditional approaches such as re-feeding and/or supplementing dietary calories and proteins may resolve the issue. However, since CC differs from starvations, to date, single modality therapies with traditional applied nutritional regimens have failed to demonstrate efficacy in improving weight gain, including gain in lean body mass in patients diagnosed with CC. The average caloric deficit in weight losing patients as with CC observed by us and other teams is approximately $250-400 \mathrm{kcals} / \mathrm{d}$. The average supplementation of 1 calorie/mL supplements have not shown to improve nutritional status of patients on chemotherapy $[35,36]$. However, recent studies using a more calorie dense $(1.5 \mathrm{kcals} / \mathrm{mL})$ and higher protein supplementation have suggested that at least weight stabilization can be achieved [37••]; although, improvements in lean body mass has not been observed in these studies. Our preliminary studies have also demonstrated that high calorie and high protein supplemental (calories/proteins dense) feedings result in substitution of regular meals and thus a reduction of caloric and protein intake in this patient population who experience early satiety. However, since most cachectic patients also exhibit symptoms of anorexia, and irregular feeding patterns, it may be important to make available to them a 
variety of dense high caloric and high protein supplements to prevent intake deficits and at a minimum stabilize weight loss.

- Other strategies include increasing physical activity, aerobic and anaerobic, as a non-pharmacological means to improve muscle and bone mass $[37 \bullet \bullet, 38 \bullet \bullet, 39]$. Although the rationale to potentially improve lean body mass by increasing physical activity is sound, patients with initial symptoms of CC, specifically during cancer treatment, report treatment-related fatigue and respiratory distress in addition to physical and time limitations and therefore demonstrate poor compliance. These symptoms are accelerated in the elderly who have relatively lower steroid hormone levels as a result of "hormonal aging," lowered physical activity, as a result of treatment-related fatigue that has been demonstrated to contribute to lowered bone mineral density and muscle mass. These age-related conditions may thus be exacerbated, resulting in falls, fractures, and general muscle weakness [33]. However, recent studies have been more encouraging and have demonstrated improvements in fatigue scores with multimodal exercise interventions [39] during chemotherapy.

- In summary, based on our work and that of others, it is clear that the most prominent feature of cachexia was that it is a condition, unlike cancer anorexia, non-responsive to traditional treatment approaches alone. Although these strategies to improve appetite, intake of nutrients and lean body mass (physical activity) have a scientific rationale and merit evaluation, studies have continued to demonstrate that reversal or control of the loss in lean body mass or skeletal and visceral proteins are difficult to achieve in CC.

\section{Pharmacological treatment}

- Since anorexia is a key component of CC, current strategies are to improve appetite by using appetite stimulants to ensure adequate intake of nutrients. Pharmacological interventions with appetite stimulants [5, 20-25], nutrient supplementation [26, 27], 5-HT3 antagonists [28, 29] and Cox-2 inhibitors [30••] have been utilized to treat CC. Although anabolic steroids and Cox-2 inhibitors have been used to treat anorexia-cachexia related to other muscle-wasting diseases such as AIDS, the use of these agents in principle are contraindicated in patients with cancer due to its growth promoting potential as well as potential cytotoxic therapy-agent interactions.

- Megesterol acetate (MA), a synthetic derivative of progestogens is commonly used to treat cancer anorexia [20-24]. The potential mechanism of action of MA is believed to involve stimulation of appetite by both direct and indirect pathways affecting the metabolism and production of catabolic cytokines [24]. MA has been refined to be more bioavailable as a nanocrystal oral suspension, significantly shortening the time to clinical response. Although appetite and weight increases have been observed with MA, there have been neither significant increase in meaningful markers of skeletal and visceral proteins nor do underlying metabolic abnormalities seen in CC.20-24. Side effects of MA include thromboembolism at high doses, transient adrenal insufficiency, edema, and central nervous system effects (confusion, headaches, dizziness, and sleep disturbances). Progesto- 
gens are also associated with a reduction in steroid hormones which results in decline in muscle mass, and an increase in fat mass [40]. These side effects in addition to lack of efficacy to increase lean body mass has tempered the routine use of MA for treatment of cancer anorexia [24].

\section{Cannabinoids}

- Other common agents include Dronabinol, a cannabis derivative, demonstrated to enhance appetite and mood in cancer patients $[25,41]$. Although the effectiveness as an appetite stimulant has been evaluated in AIDS-related wasting disease, the pharmacokinetics of this agent in cancer patients on active treatment has never been examined. In addition, because of its psychoactive side effects, difficulty in dose titration to optimize benefit, minimize side effects like dizziness, dysphoria, depression, hallucinations, alteration in psychomotor function and paranoia, time to response and long-term effects when taken orally limits the use of this agent for all cancer patients with symptoms of cancer anorexia [41]. In addition, mood alterations secondary to drug can be confused with dementia, especially in subjects who have never used cannabinoids in the past. In spite of limited data on the efficacy of cannabinoids to improve underlying metabolic abnormalities seen in CC, clinicians find it potentially useful for adjuvant treatment in palliative care to improve appetite in cancer patients with anorexia.

- Mirtazapine (Remeron, Avanza, Zispin) is a tetracyclic antidepressant used primarily in the treatment of depression, which may be a major reversible contributor of lack of appetite and weight loss [28, 29]. However, as with most appetite stimulants, have not demonstrated efficacy in the cancer patient with CC. Common side effects of mirtazapine are dizziness, blurred vision, sedation, somnolence, malaise/ lassitude, increased appetite and subsequent weight gain, dry mouth, and constipation.

- In summary, appetite stimulants have been shown to ameliorate or slow anorexia and unintentional weight loss in other populations but have not shown promise in increasing lean body mass or markers of protein metabolism in the cachectic cancer patient. Randomized clinical trials with appetite stimulants, supplementation with proteins, calories, and antioxidants although produce some stabilization in weight loss, and was not contraindicated in most cancer patients on treatment, they have failed to demonstrate success in reversing the metabolic abnormalities seen in CC. It is now clear that in addition to timely nutritional approaches, interventions based on a clear understanding of the mechanism of CC using validated markers relevant to the underlying metabolic abnormalities implicated in CC are much needed. 


\section{Class of drugs (if applicable)}

- There are currently no drugs approved for use to treat cancer cachexia.

\section{Surgery}

- There are no surgical options for the treatment of cancer cachexia.

Other therapies

- There are no other therapies for the treatment of cancer cachexia.

\section{Emerging therapies}

- Eicosapentaenoic acid (EPA) is an $n-3$ fatty acids and an essential fatty acid that cannot be synthesized completely by mammalian tissue and has to be consumed in the diet. These polyunsaturated fats are present in fish oils such as cod liver, sardine, and salmon oil. There is no established Dietary Reference Intake for $n-3$ fatty acids; yet, the adequate intake (AI) is set at 1.6 and $1.1 \mathrm{~g} / \mathrm{d}$ for men and women, respectively. While intake in the United States occurs at much lower than the proposed AI and no signs of deficiency are observed, the AI is proposed to provide optimal health benefits associated with consuming $n$-3 fatty acids [42]. Evidence from laboratory and clinical studies has demonstrated that EPA has antitumor and anticachectic effects. Initial studies using animal models have demonstrated that $\mathrm{NFkB}$ is upregulated in CC increasing proteolysis and inducing apoptosis in myotubes $[43,44]$. Several recent laboratory studies have shown that EPA may attenuate protein degradation, by preventing NFkB accumulation in the nucleus [43, 44]. Administration of $n-3$ fatty acids and EPA capsules or supplements with EPA has been shown to be associated to weight stabilization, gain in lean body mass, and improvement in quality of life markers in weight losing patients with advanced pancreatic cancer. A tumor product, PIF is produced by cachexia-inducing murine and human tumors and initiates muscle protein degradation directly through activation of the proteasome pathway. The action of PIF is blocked by eicosapentaenoic acid (EPA), which has been shown to attenuate the development of cachexia in pancreatic cancer patients [10,11]. Clinical trials to date have produced mixed results potentially attributed to interventions in later stage of disease, inadequate monitoring, poor compliance, and selection of primary outcome markers such as weight-which has been shown to be confounded by hydration, cytotoxic therapies, and acute disease status and serum cytokines, all of which may have limited value in evaluating interventions in patients with CC, especially those patients on active treatment. To our knowledge, $n-3$ fatty acid supplements have never been evaluated pro-actively in the early stages of CC for the prevention of progressive protein loss in CC populations with a relatively better prognosis or those populations who could potentially demonstrate significant improvement in treatment outcomes, functional status, and quality of life. Based on our preliminary observations and that of others, we hypothesize that by selective targeting of proteasome activity by a standardized dose of $n-3$ 
fatty acids early in the diagnosis of $C C$, will alter metabolic abnormalities by downregulating NFkB, modulate PIF and inflammatory response thus preventing the breakdown of myofibrillar proteins resulting in increasing serum protein markers, lean body mass and physical function. We completed a pilot trial of 36 subjects to evaluate the safety and effectiveness of administering 4-g Lovaza ${ }^{\circledR}$ (omega-3acid ethyl esters) for a 6-week period, to cancer patients ( $>65 \%$ lung), with early stages of CC (unplanned weight loss of 5\% of their body weight within the past 3 months) and observed changes in markers of toxicity, visceral and skeletal protein status, nutritional intake, cytokines, functional status and physical activity. Lovaza ${ }^{\circledR}$, is an FDAapproved agent (GlaxoSmithKline IND \# 45,998) that provides prescription-only potency and purity, contains within each 1-g Lovaza ${ }^{\circledR}$ (omega-3-acid ethyl esters) gel capsule 90\% omega-3-acid ethyl esters, $84 \%$ eicosapentanoic acid (EPA) and docosahexaenoic acid (DHA) ethyl esters. Subjects were predominantly Caucasian and male with $63 \%$ diagnosed with lung cancer. 31\% reported alcohol use of at least one drink weekly and $60 \%$ of subjects reported history of smoking with $8.6 \%$ reporting that they currently smoke. Pill count and study agent logs indicated an average compliance of $93 \%$ of study agent and $86 \%$ compliance to nutritional intake recommendations. Anthropometrics measurements such as height, weight, body mass index (BMI), triceps skinfold and mid-arm muscle circumference measurements remained stable with no reductions observed in these markers during the 6-week intervention period. Slight increase in calories, carbohydrates and fat intake was observed and stable intake of proteins noted over the 6 week intervention period. Most importantly, we observed statistically significant increases in serum albumin $(P<0.0001)$, moderately significant increase in serum transferrin $(P<0.07)$ (Table 1), improvement in functional status and physical activity with no toxicity related to the study drug at this dose. Although serum levels of cytokines TNF- $\alpha$ (Cohen's $d$ 0.248) progressively increased and IL6 decreased slightly (Cohen's $d-0.019$ ), since patients were on active treatment with cytotoxic agents, these were difficult to interpret.

- In addition, we explored if treatment with Lovaza ${ }^{\circledR}$ at a dose of $4 \mathrm{~g} / \mathrm{d}$ results in inhibition of proteasome activity in pre and post-treatment serum samples from the patients in our clinical trial. Venous blood was collected from subjects at baseline and at 6 weeks post intervention into heparin-containing tubes and proteasome assay was carried out using these samples using Proteasome ELISA Kit according to manufacturer's instructions (Proteasome ELISA Kit, ENZO Life Sciences, Cat\# PW0575). Complete pre and post-treatment serum samples were available for 14 subjects for these studies Inhibition of proteasome activity in serum samples post treatment was calculated and plotted based on proteasome activity of pre-treatment samples. Results demonstrated that proteasome activity was inhibited in nine out of fourteen patients (64\%) in the range of $6 \%-29 \%$ (Fig. 1). Based on the evidence from these preliminary studies by our group, it is evident that indeed standardized supplementation with $n-3$ fatty acids appear to be promising agents that may attenuate protein degradation by targeting the proteasomes, improve skeletal and visceral proteins, functional status and physical activity and have enough evidence to warrant use in well powered, randomized clinical trials to further examine its safety, efficacy, and validate the mechanisms by which they modulate the metabolic abnormalities associated with CC. 


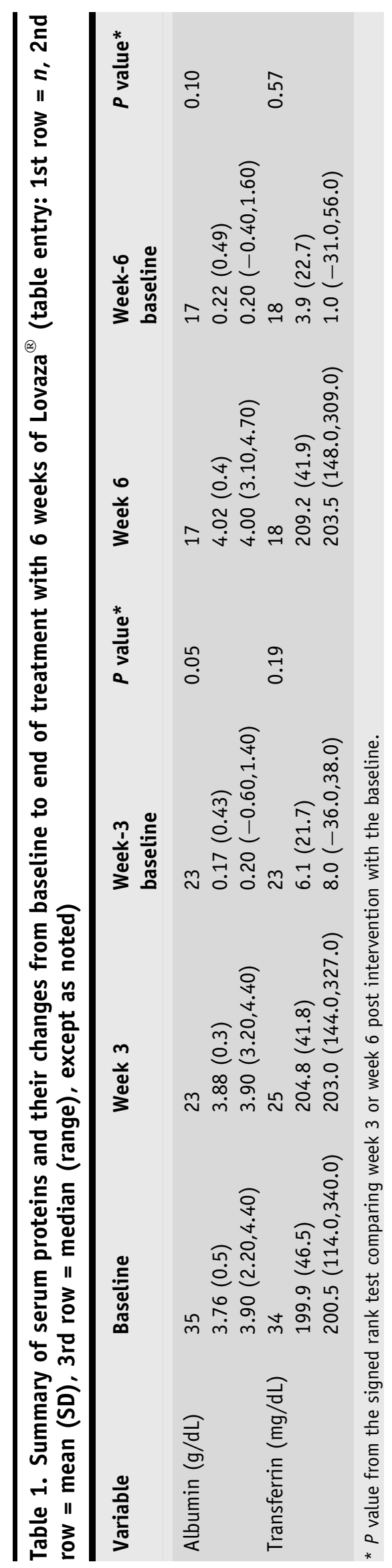




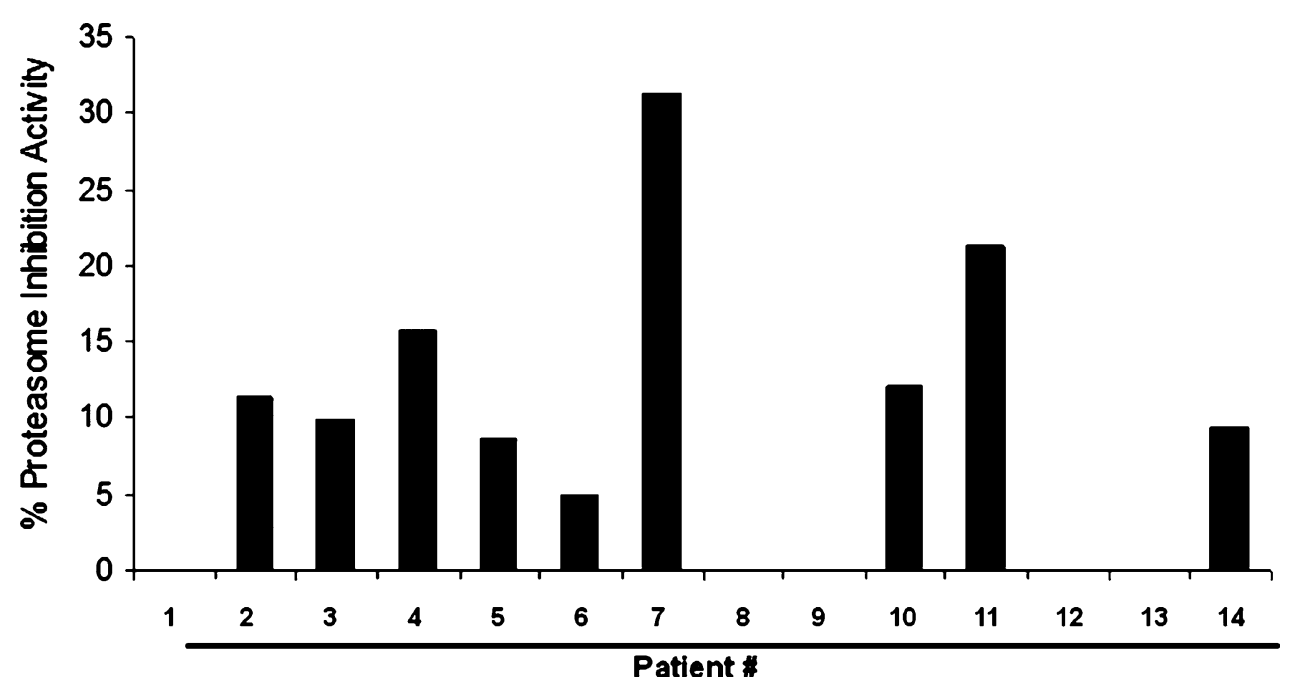

Figure 1. Proteasome inhibitory activity in serum samples of 14 patients with early symptoms of cancer cachexia treated with $n-3$ fatty acid supplements.

- Unlike previous studies that reported mixed results with interventions in pancreatic cancer or late stage cancer patient populations [43-46••] the aim of this study was to examine the initial safety, efficacy and potential mechanism by which we can prevent progression of CC early in the diagnosis with a standardized preparation of $n-3$ fatty acid supplements in a population of cancer patients with a relatively better prognosis. We have, in addition, made an important distinction between CC and anorexia (where appetite and weight are the primary concern). This is an important distinction that allows us to focus on the intermediate biomarkers of these underlying metabolic disorders, specific to CC in addition to the symptoms of cancer anorexia. Furthermore, we utilized a novel, molecular mechanism-based approach to treating CC, which is important to move this field of research further.

- Clearly, there is a need to use a molecular mechanism-based approach to identify potential agents for the treatment of CC. It may be critical to examine the potential to intervene in pathways understood at the molecular level to improve appetite, modify metabolic alterations that contribute to wasting, and downregulate transcription factors or cytokine-induced events to treat CC. Based on the evidence from laboratory and clinical trials, it is evident that EPA is a promising agent that may attenuate protein degradation by targeting the proteasomes, and have enough evidence to warrant use in clinical trials to examine its efficacy for the treatment of CC. Although improving the underlying metabolic abnormalities observed in CC for all patients may not be possible, the aim must be to intervene in the early stages of this disease to stabilize CC and prevent or delay further decline. Both survival and quality of survival are important outcomes. Thus, developing and refining current strategies to additionally counteract the symptom clusters of CC using multimodal interventions (nutritional supplementation, appetite stimulants, and physical activity regimen) to improve function and quality of life of cancer patients continue to be crucial. 
Acknowledgments The research study was funded partly by GlaxoSmithKline, NC. We thank Xiuhua Zhao for her assistance with the statistical analysis and Claire Jordan for the preparation of the manuscript.

Open Access This article is distributed under the terms of the Creative Commons Attribution Noncommercial License which permits any noncommercial use, distribution, and reproduction in any medium, provided the original author(s) and source are credited.

Publications To date, the results of this study in part or as a whole have not been published elsewhere.

Disclaimers None

\section{References and Recommended Reading}

Papers of particular interest, published recently, have been highlighted as:

- Of importance

•• Of major importance

1. Ross PJ, Ashley S, Norton A, et al.: Do patients with weight loss have a worse outcome when undergoing chemotherapy for lung cancers? Br J Cancer 2004, 90:1905-1911.

2. Jatoi A: Weight loss in patient with advanced cancer: effects, causes, and potential management. Curr Opin Support Palliat Care 2008, 2(1):45-48.

3. Evans WJ, Morley JE, Argilés J, et al.: Cachexia: a new definition. Clin Nutr 2008, 27(6):793-799.

A classic manuscript that proposes the definition and comprehensive discussion of the CC syndrome.

4. Behl D, Jatoi A: Pharmacological options for advanced cancer patients with loss of appetite and weight. Expert Opin Pharmacother 2007, 8(8):1085-1090.

5. Hall W, Christie M, Currow D: Cannabinoids and cancer: causation, remediation, and palliation. Lancet Oncol 2005, 6(1):35-42.

6. Maltoni M, Fabbri L, Nanni O, et al.: Serum levels of tumor necrosis factor alpha and other cytokines do not correlate with weight loss and anorexia in cancer patients. Support Care Cancer 1997, 5(2): 130-135

(comments in Support Care Cancer. 1997;5(5):422-3).

7. Barton BE: IL-6-like cytokines and cancer cachexia: consequences of chronic inflammation. Immunol Res 2001, 23(1):1-58.

8. Mantovani G, Maccio A, Mura L, et al.: Serum levels of leptin and proinflammatory cytokines in patients with advanced-stage cancer at different sites. $J \mathrm{Mol}$ Med. 2000, 78(10):554-561.

9. Deans DA, Wigmore SJ, Gilmour H, et al.: Expression of the proteolysis-inducing factor core peptide mRNA is upregulated in both tumour and adjacent normal tissue in gastro-esophageal malignancy. $\mathrm{Br} \mathrm{J}$ Cancer 2008, 98(1):242

Author reply 243.

10. Wigmore SJ, Todorov PT, Barber MD, et al.: Characteristics of patients with pancreatic cancer expressing a novel cancer cachectic factor. Br J Surg. 2000, 87(1):53-58.

11.• Tisdale MJ: Catabolic mediators of CC. Curr Opin Support Palliat Care 2008, 2(4):256-261.

This review is comprehensive review that compares the catabolic actions of tumour necrosis factor-alpha (TNF-alpha) and proteolysis-inducing factor (PIF) and their involvement in human cancer cachexia.

12.•• Deans C, Wigmore SJ: Systemic inflammation, cachexia and prognosis in patients with cancer. Curr Opin Clin Nurs Metal Care 2005, 8(3):265-269.

This excellent review summarizes current thinking relating to inflammation, cachexia and prognosis in cancer patients, with particular emphasis on studies relating to recent therapeutic advances

13. Ciechanover A, Orian A, Schwartz AL: The ubiquitinmediated proteolytic pathway: mode of action and clinical implications. J Cell Biochem Suppl. 2000, 34:40-51.

This article reviews the enzymatic cascade involved in ubiquitin-mediated degradation, describes some of the structural motifs identified by the conjugating machinery, and summarize recent developments in the involvement of the system in the pathogenesis of selected disease states, including CC.

14. Ghosh S, Karin M: Missing pieces in the NF-kappaB puzzle. Cell 2002, 109(Suppl):S81-S96.

15. Chen S, Fribley A, Wang CY: Potentiation of tumor necrosis factor-mediated apoptosis of oral squamous cell carcinoma cells by adenovirus-mediated gene transfer of NF-kappaB inhibitor. J Dent Res. 2002, 81:98-102.

16. Mitch WE, Price SR: Transcription factors and muscle cachexia: is there a therapeutic target? Lancet 2001, 357(9258):734-735.

17. Hochstrasser $M$ : Origin and function of ubiquitinlike proteins. Nature 2009, 458(7237):422-429.

18. Voges D, Zwickl P, Baumeister W: The 26S proteasome: a molecular machine designed for controlled proteolysis. Annu Rev Biochem. 1999, 68:1015-1068. 
19. Loprinzi CL, Michalak JC, Schaid DJ, et al.: Phase III evaluation of four doses of megestrol acetate as therapy for patients with cancer anorexia and/or cachexia. J Clin Oncol. 1993, 11(4):762-767.

20. Lynch GS, Schertzer JD, Ryall JG: Therapeutic approaches for muscle wasting disorders. Pharmacol Ther 2007, 113(3):461-487.

21. Karcic E, Philpot C, Morley JE: Treating malnutrition with megestrol acetate: literature review and review of our experience. J Nutr Health Aging 2002, 6(3):191-200.

22. Morley JE: Orexigenic and anabolic agents. Clin Geriatr Med 2002, 18(4):853-866.

23. Femia RA, Goyette RE: The science of megestrol acetate delivery: potential to improve outcomes in cachexia. BioDrugs 2005, 19(3):179-187.

24. Walsh D, Nelson KA, Mahmoud FA: Established and potential therapeutic applications of cannabinoids in oncology. Support Care Cancer 2003, 11(3):137143.

25. Ovesen L, Allingstrup L, Hannibal J, et al.: Effect of dietary counseling on food intake, body weight, response rate, survival, and quality of life in cancer patients undergoing chemotherapy: a prospective, randomized study. J Clin Oncol. 1993, 11:2043-2049.

26. Deans DA, Tan BH, Wigmore SJ, et al.: The influence of systemic inflammation, dietary intake and stage of disease on rate of weight loss in patients with gastroesophageal cancer. Br J Cancer 2009, 100(1):63-69.

27. Riechelmann RP, Burman D, Tannock IF, et al.: Phase II trial of mirtazapine for cancer-related cachexia and anorexia. Am J Hosp Palliat Care 2010, 27(2):106-110.

28. Kast RE, Foley KF: Cancer chemotherapy and cachexia: mirtazapine and olanzapine as 5-HT3 antagonists with good antinausea effects. Eur J Cancer Care (Engl). 2007, 16(4):351-354.

29. Mantovani G, Madeddu C: Cyclooxygenase-2 inhibitors and antioxidants in the treatment of cachexia. Curr Opin Support Palliat Care 2008, 2(4):275-281.

30.•Bozzetti F, Mariani L: Defining and classifying CC: a proposal by SCRINIO Working Group. JPEN J Parenter Enteral Nutr 2009, 33(4):361-367.

31. Blum B, Omlin A, Fearon K, et al.: European Palliative Care Research Collaborative. Evolving classification systems for CC: ready for clinical practice? Support Care Cancer 2010, 18(3):273-279.

32. McMillan DC: Systemic inflammation, nutritional status and survival in patients with cancer. Curr Opin Clin Nutr Metab Care 2009, 12(3):223-226.

33. Morley JE: Anorexia and weight loss in older persons. J Gerontol A Biol Sci Med Sci. 2003, 58(2):131-137.

$34 . \bullet$ Bosaeus I: Nutritional support in multimodal therapy for cancer cachexia. Support Care Cancer 2008, 16(5):447-451.

This review provides a pragmatic approach to the treatment of cachexia, stating the goal must be to stabilise cachexia and prevent or delay further decline and established the need to evaluate strategies to counteract both hypermetabolism and reduced dietary intake and the importance of improving not only survival but function and quality of life of cancer patients.
35. Couch M, Lai V, Cannon T, et al:: Cancer cachexia syndrome in head and neck cancer patients: part $\mathrm{I}$. Diagnosis, impact on quality of life and survival, and treatment. Head Neck 2007, 29(4):401-411.

36. Fearon KC, von Meyenfeldt M, Moses AG, et al.: Effect of a protein and energy dense n-3 fatty acid enriched oral supplement on weight and lean tissue in CC: a randomized double blind trial. Gut 2003, 52(10):1479-1486.

37.• Fearon KC: Cancer cachexia: developing multimodal therapy for a multidimensional problem. Eur J Cancer 2008, 44(8):1124-1132.

This review provides a comprehensive evidence-base and makes a case for multimodal approaches for the design of complex intervention studies in the management of cancer cachexia.

38.• Al-Majid S, Waters H: The biological mechanisms of cancer-related skeletal muscle wasting: the role of progressive resistance exercise. Biol Res Nurs 2008, 10(1):7-20.

This article discusses several cancer-related skeletal muscle wasting mechanisms and proposes how physical activity might attenuate muscle wasting by counteracting some of these mechanisms.

39. Karcic E, Philpot C, Morley JE: Treating malnutrition with megestrol acetate: literature review and review of our experience. J Nutr Health Aging 2002, 6(3):191-200.

40. De Petrocellis L, Melck D, Bisogno T, Di Marzo V: Endocannabinoids and fatty acid amides in cancer, inflammation and related disorders. Chem Phys Lipids 2000, 108(1-2):191-209.

41. United States Department of Health \& Human Services, Statistics, (USDHHS), March 4, 2002.

42. Fearon KC, Van Meyenfeldt MF, Moses AG, et al.: Effect of a protein and energy dense N-3 fatty acid enriched oral supplement on loss of weight and lean tissue in CC: a randomised double blind trial. Gut 2003, 52(10):1479-1486.

43. Dewey A, Baughan C, Dean T, et al.: Eicosapentaenoic acid (EPA, an omega-3 fatty acid from fish oils) for the treatment of cancer cachexia. Cochrane Database Syst Rev 2007, 24(1):CD004597.

44. Hamerman D: Molecular-based therapeutic approaches in treatment of anorexia of aging and cancer cachexia. J Gerontol A Biol Sci Med Sci 2002, 57(8):M511-M518.

45.••Mantovani G, Macciò A, Madeddu C, et al.: Randomized phase III clinical trial of five different arms of treatment for patients with cancer cachexia: interim results. Nutrition 2008, 24(4):305-313.

This is a phase III randomized study which includes all the agents currently used to treat CC and examines several safety and efficacy endpoints of cancer cachexia: lean body mass (LBM), resting energy expenditure (REE), fatigue; and relevant secondary endpoints: appetite, quality of life, grip strength, Glasgow Prognostic Score (GPS) and proinflammatory cytokines.

46. Wigmore SJ, Ross JA, Falconer JS, et al: : The effect of polyunsaturated fatty acids on the progress of cachexia in patients with pancreatic cancer. Nutrition 1996, 12(1 Suppl):S27-S305. 